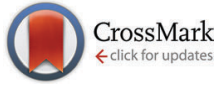

Cite this: Chem. Commun., 2014, 50, 13742

Received 14th August 2014, Accepted 8th September 2014

DOI: $10.1039 / c 4 c c 06371 f$

www.rsc.org/chemcomm

\section{Synthesis and properties of DNA oligonucleotides with a zwitterionic backbone structure $\dagger$}

\author{
Boris Schmidtgall, ${ }^{\text {ab }}$ Anatol P. Spork, ${ }^{\mathrm{b}}$ Falk Wachowius, ${ }^{\mathrm{c}}$ Claudia Höbartner ${ }^{\mathrm{c}}$ and \\ Christian Ducho*ad
}

The nucleosyl amino acid (NAA)-modification of oligonucleotides is introduced, which enables the preparation of oligonucleotides with zwitterionic backbone structures. It is demonstrated that partially zwitterionic NAA-modified DNA oligonucleotides are capable of duplex formation with native polyanionic counterstrands and show retained sensitivity towards base-pairing mismatches.

Oligonucleotides represent promising biomedical agents for antigene, antisense or RNA interference applications. ${ }^{1}$ However, due to their high polarity hindering cellular uptake and their limited stability towards nucleases, chemical or enzymatic modifications of nucleic acid structures ${ }^{2,3}$ are an essential prerequisite for their biomedical application.

One structural feature of nucleic acids which has attracted significant interest is their polyanionic backbone resulting from the linking phosphate diesters. ${ }^{4}$ The polyanionic nature of nucleic acids largely contributes to their limited biomedical applicability, and hence, several chemical modifications have aimed at the manipulation of the backbone's charge pattern. Thus, a significant number of artificial electroneutral internucleotide linkages such as sulfone, ${ }^{5}$ triazole $^{6}$ or amide moieties $^{7-10}$ have been studied. Peptide nucleic acid (PNA) represents probably the most striking example of an electroneutral nucleic acid analogue. ${ }^{11,12}$ However, fully electroneutral nucleic acid analogues often show low water solubility and are prone to aggregate formation.

\footnotetext{
${ }^{a}$ University of Paderborn, Department of Chemistry, Warburger Str. 100, 33098 Paderborn, Germany

${ }^{b}$ Georg-August-University Göttingen, Department of Chemistry, Institute of Organic and Biomolecular Chemistry, Tammannstr. 2, 37077 Göttingen, Germany

${ }^{c}$ Max-Planck-Institute for Biophysical Chemistry, Am Fassberg 11, 37077 Göttingen, Germany

${ }^{d}$ Saarland University, Department of Pharmacy, Pharmaceutical and Medicinal Chemistry, Campus C2 3, 66123 Saarbrücken, Germany.

E-mail: christian.ducho@uni-saarland.de

$\dagger$ Electronic supplementary information (ESI) available: Synthesis of phosphoramidites and oligonucleotides, NMR spectra, analytical data of oligonucleotides, $T_{\mathrm{m}}$ data and CD spectra of oligonucleotide duplexes. See DOI: 10.1039/c4cc06371f
}

The apparent conclusion to overcome such problems arising from electroneutral backbone structures is the introduction of positively charged moieties into the backbone, thus resulting in zwitterionic structures. Several examples for the attachment of positively charged units, often linked to the $2^{\prime}$-hydroxy group or the nucleobase, have been reported, ${ }^{13-15}$ but in these cases, the phosphate-derived backbone has been kept intact. Thus, even though some of the resultant oligonucleotide analogues showed promising properties, this strategy leads to densely charged oligomers as the positive charge is an additional feature in these cases. An alternative approach can be pursued by replacing some of the phosphate moieties with positively charged groups as linker units, ${ }^{16}$ thus retaining the overall number of charges in the oligomer. Consequently, a nucleic acid analogue with such a 'real' zwitterionic backbone may be expected to be less polar than the aforementioned congeners with attached cationic units.

Due to electrostatic attraction, the presence of alternating charge patterns in zwitterionic backbones may harm fundamental properties of nucleic acids though. Benner has hypothesised that a uniform charge pattern in the backbone is not only crucial to prevent longer nucleic acid strands from folding, but that the repeating anionic motifs are also important for the molecular recognition of two native nucleic acid strands with complementary base sequences. ${ }^{17,18}$ It is therefore of major importance to study whether oligonucleotides with zwitterionic backbones can still form stable duplex structures and retain base pairing fidelity.

Overall, only few positively charged artificial internucleotide linkages have been reported, ${ }^{16}$ and consequently, oligonucleotides with zwitterionic backbones have not been extensively studied yet. Bruice et al. have replaced the phosphate unit with either a guanidine ${ }^{19}$ or with an $S$-methylthiourea ${ }^{20}$ group. The use of these moieties for the preparation of partially zwitterionic oligonucleotides ('chimera,16) was only studied with few oligonucleotides though, and the results were not fully conclusive. ${ }^{21,22}$ Letsinger et al. have developed phosphoramidate linkages in which positively charged groups (namely amines) were connected 

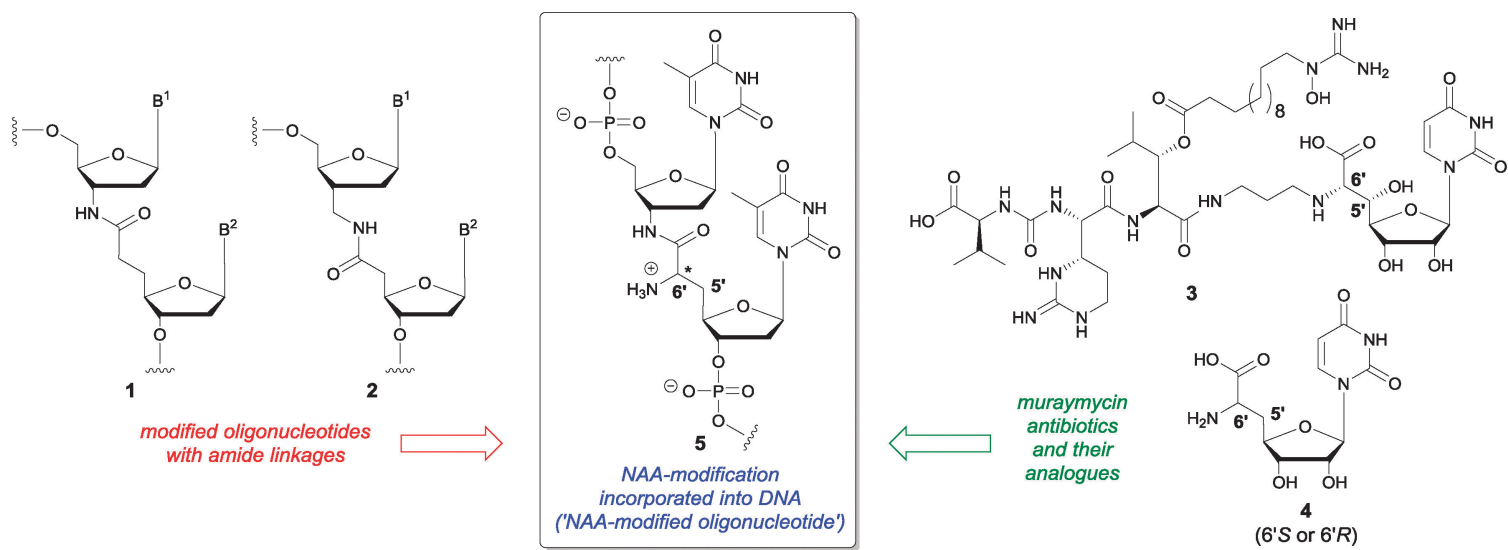

Fig. 1 Design concept of nucleosyl amino acid (NAA)-modified oligonucleotides.

to the backbone via alkyl linkers. ${ }^{23}$ They could thus obtain even fully zwitterionic DNA, but due to the conformational flexibility of the positively charged moieties, one cannot rule out interactions with the phosphate groups which would be less likely if the positively charged moieties were more rigidly fixed at the internucleotide linkage sites.

We have now conceived a novel artificial internucleotide linkage which enables the preparation of zwitterionic nucleic acid analogues. Two structural motifs contributed to the design of this new internucleotide unit (Fig. 1): (i) established amide internucleotide linkages such as $\mathbf{1}$ and $\mathbf{2}^{7-10}$ and (ii) the nucleoside moiety of muraymycin nucleoside antibiotics (e.g. muraymycin A5 3) and its 5'-deoxy analogues 4. Muraymycins represent a promising class of natural products with antimicrobial activity. ${ }^{24}$ In the course of our synthetic investigations on muraymycins, ${ }^{25-27}$ we have previously reported on the efficient stereoselective preparation of protected forms of $\mathbf{4}$ and have coined the term nucleosyl amino acids (NAAs) for these types of compounds. ${ }^{28,29}$ However, if one merges the structural principles of $\mathbf{4}$ with an amide internucleotide bridge of type 1, an oligonucleotide modification of type $\mathbf{5}$ ('NAA-modification') is obtained. The amino group of the NAA-modification is expected to be positively charged at physiological $\mathrm{pH}$ values. The configuration of the stereocenter within the NAA-linkage is anticipated to influence the spatial orientation of this cationic motif. In contrast to Letsinger's approach (vide supra), the NAA-motif provides a positive charge in a conformationally more fixed position which is in immediate vicinity to the position of the negative charge in native phosphate linkages. The NAAmodification therefore enables the introduction of positive charges at specific sites of an otherwise phosphate-linked oligoanionic nucleic acid, thus representing a novel system providing (partially) zwitterionic DNA analogues.

For the automated solid-phase synthesis of stereoisomerically pure NAA-modified oligonucleotides, 'dimeric' phosphoramidite building blocks containing the protected NAA-motif with either $\left(6^{\prime} S\right)$ - or $\left(6^{\prime} R\right)$-configuration were prepared (see ESI $\left.\dagger\right)$. We decided to initiate our studies with the introduction of NAA-modifications at T-T motifs. Analogous NAA-modified X-T motifs (with X = A, C, G) will then later be accessible using our synthetic route (see ESI $\dagger$ ).
The obtained 'dimeric' phosphoramidites were used for the automated synthesis of 24 different DNA oligonucleotides bearing one, two or four NAA-modifications at various positions and thus having partially zwitterionic backbone structures. After oligonucleotide synthesis and base-mediated cleavage from the solid support, the desired full-length products were purified and isolated. The fully characterised modified oligonucleotides (see ESI $\dagger$ ) are classified into structural types of sequences 6, 7 and 8 (Fig. 2). All modified

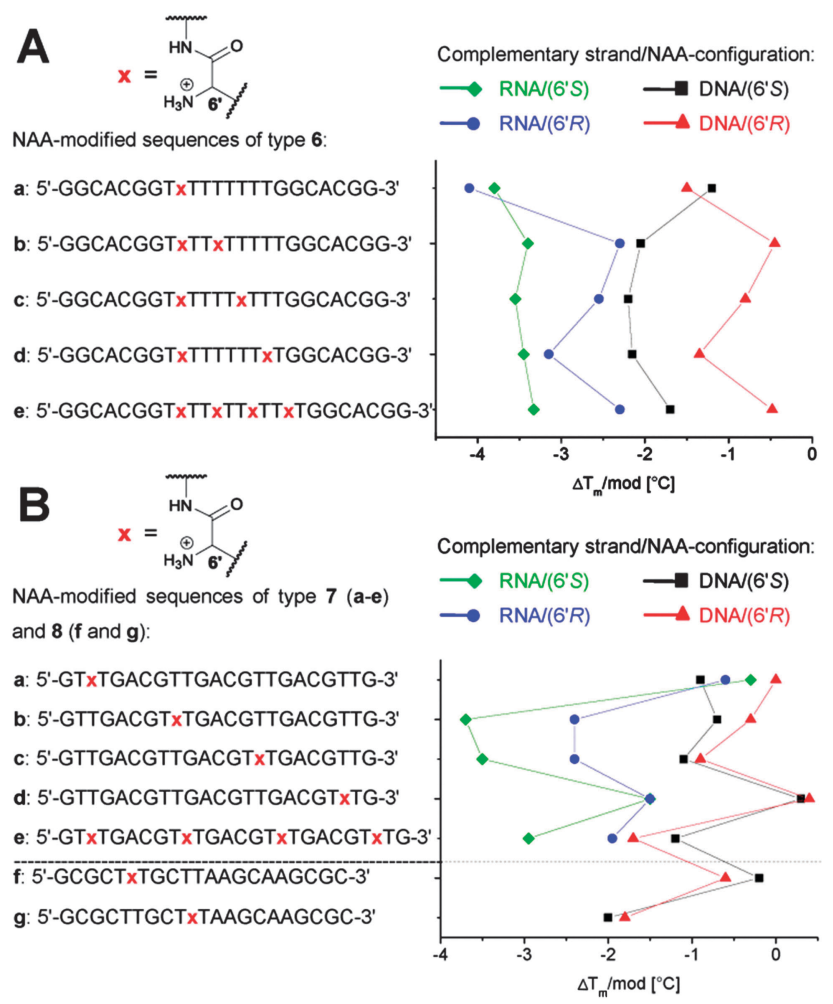

Fig. 2 Stability of duplexes formed with NAA-modified DNA oligonucleotides of types $\mathbf{6}$ (A) as well as $\mathbf{7}$ and $\mathbf{8}$ (B). Note that $\mathbf{8}$ ( $\mathbf{f}$ and $\mathbf{g}$ in B) is selfcomplementary. Sites of NAA-modifications $=x$, all other linkages were native phosphates. Complementary strand: native DNA or native RNA with fully matching sequences (for $\mathbf{6}$ and 7 ). Values in the diagram: alterations of duplex $T_{m}$ values per NAA-modification $\left(\Delta T_{m} / \mathrm{mod}\right)$ in comparison to native duplexes without NAA-modifications. For full data see Tables S3 to S7 (ESI†). 
oligonucleotides formed duplexes with complementary DNA and RNA oligonucleotides at physiologically relevant salt concentrations. The stability of these duplexes was studied by UV-monitored thermal denaturation providing melting temperatures ( $T_{\mathrm{m}}$ values).

Modified DNA oligonucleotides of type $\mathbf{6}$ were designed in order to test if the distance between two NAA-modifications within a strand exerts an influence on the structure and stability of nucleic acid duplexes (Fig. 2A). The results indicate that all duplexes containing one or more NAA-modifications in one strand were moderately destabilised in comparison to their unmodified congeners. NAA-modified DNA-RNA hybrids were destabilised more strongly ( $\sim 2$ to $4{ }^{\circ} \mathrm{C}$ per mod) than DNA/DNA duplexes ( $\sim 0.5$ to $2{ }^{\circ} \mathrm{C}$ per mod). Also, a slight difference in thermal stability $\left(\sim 1{ }^{\circ} \mathrm{C}\right.$ per mod) between duplexes containing the $\left(6^{\prime} S\right)$-NAA- and the $\left(6^{\prime} R\right)$-NAA-modification was observed in nearly all experiments. However, no evidence was found for a relationship between the distance of two NAA-modifications (in the case of sequences b-d, Fig. 2A) and the duplex stability. Remarkably, DNA/DNA duplexes with sequences of type e did not display a more pronounced destabilisation although e contained four consecutive NAA-motifs, i.e. a zwitterionic backbone segment spanning over ten base pairs (with the charge pattern $-/+1-1+/-1+1-1+/-)$. Interestingly, the DNA/DNA duplex containing the $\left(6^{\prime} R\right)$-isomer of sequence e showed a $\Delta T_{\mathrm{m}} / \mathrm{mod}$ value of only $-0.5{ }^{\circ} \mathrm{C}$, indicating that such a significantly modified DNA structure can display nearly the same melting stability as its native congener. For the discussion of all reported $T_{\mathrm{m}}$ values, it should be taken into account that the experimental precision of $T_{\mathrm{m}}$ values is estimated to be around $\pm 1{ }^{\circ} \mathrm{C},{ }^{30}$ translating into $\Delta T_{\mathrm{m}} / \bmod$ values, for example, as low as $\pm 0.25{ }^{\circ} \mathrm{C}$ for duplexes containing four NAA-modifications.

In a second set of experiments, several sequences of structural type 7 were studied to investigate the correlation between the position of the modification and the duplex stability. In general, it was found that internal NAA-modifications cause a stronger destabilisation compared to those in proximity to the $3^{\prime}$ - or the $5^{\prime}$-end (Fig. 2B). As for sequences 6, the decrease in duplex stability was more pronounced in the case of DNA/RNA duplexes and a similar effect of the NAA-configuration was observed. In the case of sequence e (Fig. 2B) bearing four NAA-modifications (in pronounced distance to each other though), no extraordinary drop in duplex stability was found. In addition, self-complementary palindromic sequences $\mathbf{8}$ were used to study the self-pairing between two NAA-modified DNA strands. It was thus demonstrated that two strands bearing the NAA-modification are able to form duplexes with only a moderate decrease in stability compared to the native congeners $\left(\Delta T_{\mathrm{m}} \sim 0\right.$ to $\left.-2{ }^{\circ} \mathrm{C}\right)$.

Overall, the results depicted in Fig. 2 indicate a moderate destabilisation of DNA/DNA and DNA/RNA duplexes due to the presence of NAA-modifications. DNA/DNA duplexes proved to be less destabilised by NAA-modifications than DNA-RNA hybrids. The reason for these effects might be the rigid peptide bond which fixes the torsion angle of the $\zeta$-bond, giving rise to a conformation that is less favourable for base pairing and stacking than in the case of native phosphate linkages. Apparently, the NAA-modification is better accommodated in a B-form helix than

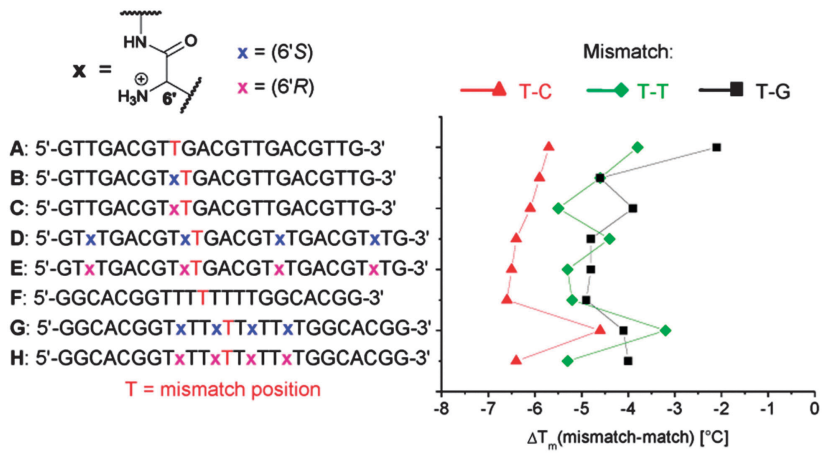

Fig. 3 Destabilisation of DNA/DNA duplexes with one NAA-modified strand caused by base-pairing mismatches. Depicted sequences formed duplexes with DNA complements bearing mismatches at the indicated position (opposite of red T). Sequences A and F: unmodified native references without NAA-modifications. Values in the diagram: alterations of duplex $T_{m}$ values resulting from the presence of the mismatch, i.e. $T_{m}$ values of mismatch-containing duplexes in comparison to similar duplexes with fully matching sequences $\left(\Delta T_{\mathrm{m}}\right.$ (mismatch-match)). For full data see Table S8 (ESI $\dagger)$

in the A-form of the DNA-RNA hybrid, thus leading to a greater loss of thermal stability in the case of DNA/RNA duplexes. However, this also implies that conformational effects can overrule effects resulting from the charge pattern of the backbone, thus questioning the supposedly crucial significance of the charges for the overall properties of nucleic acids (vide supra).

Another aspect of interest was the base-pairing specificity of the modified nucleic acids. Thus, some of the NAA-modified oligonucleotides were chosen for melting temperature experiments with DNA complements containing a mismatched base in close proximity to the modification (Fig. 3). In this context, we compared the $T_{\mathrm{m}}$ values of duplexes composed of NAAmodified oligonucleotides B-E and G, $\mathbf{H}$ (Fig. 3) and a native counterstrand having one mismatched base to corresponding duplexes derived from unmodified reference oligonucleotides $\mathbf{A}$ and $\mathbf{F}$, respectively. In general, a pronounced destabilisation of the duplexes resulting from the mismatch was retained in all NAA-modified duplexes. It can thus be concluded that the mismatch recognition of nucleic acids remains nearly untouched by the presence of NAA-modifications in the backbone regardless of their number or position. The sensitivity towards base-pairing mismatches is not overruled by the presence of positive charges in the NAA-modified backbone and the supposedly resulting partial electrostatic attraction.

We also examined the properties of NAA-modified duplexes under various ionic strengths. Melting temperature experiments at elevated concentrations of sodium chloride were performed (see Tables S9 to S12, ESI $\dagger$ ). Native oligoanionic DNA/DNA duplexes show increased $T_{\mathrm{m}}$ values at higher sodium chloride concentrations. Using their approach employing alkyllinked positively charged moieties (vide supra), Letsinger et al. have reported that a fully zwitterionic nonameric oligo-dT analogue formed a duplex with poly-dA with a $T_{\mathrm{m}}$ value independent of ionic strength, i.e. sodium chloride concentration. ${ }^{23}$ In the case of the partially zwitterionic NAA-modified DNA/DNA 
duplexes reported in this study, the increase of $T_{\mathrm{m}}$ values as a function of sodium chloride concentration was slightly less pronounced than for the respective native reference duplexes (Tables S9 and S11, ESI $\dagger$ ). This may reflect the partially zwitterionic charge pattern in the backbone of NAA-modified duplexes. To shed light on possible structural distortions of nucleic acid topology arising from the presence of zwitterionic segments in the backbone, circular dichroism (CD) spectra of all NAA-modified duplexes and of unmodified reference duplexes were recorded. All DNA/RNA and DNA/DNA duplexes showed the characteristic features of A- and B-form helices, respectively, and no significant differences between NAAmodified and unmodified native nucleic acids were observed (see $\mathrm{ESI} \dagger$ ).

In summary, typical chemical properties of nucleic acids are retained in NAA-modified DNA oligonucleotides. Despite their partial zwitterionic backbone, they are capable of forming helical duplexes (though moderate destabilisation occurs) and of recognising base-pairing mismatches. This is remarkable as the backbone structure of these analogues is quite different from native fully phosphate-linked systems. Hence, we conclude that zwitterionic segments within the backbone of nucleic acids are tolerated quite well in terms of the chemical properties of the resultant oligonucleotide analogues. However, our studies so far only provide insights into the properties (i) of shorter oligonucleotides (20- to 22-mers) with modified charge patterns and (ii) of partially zwitterionic systems. The data reported in this work provide a starting point though of more detailed future investigations. These will include studies (i) on longer NAA-modified nucleic acids, (ii) on oligonucleotides with a fully zwitterionic backbone and (iii) on NAA-modifications with mixed base sequences at the NAA-linkage site. Furthermore, we will address the interaction of NAA-modified oligonucleotides with enzymes such as nucleases and polymerases. Due to the reduced overall negative charge, we anticipate NAA-modified oligonucleotides to possibly display enhanced cellular uptake. Hence, the NAAlinkage might be a useful addition to the 'toolbox' of oligonucleotide modifications ${ }^{2,3}$ for the design of therapeutic agents. It should also be noted that the amino group of the NAA-motif might be employed for the selective covalent derivatisation of oligonucleotides with fluorophores or other chemical entities.

We thank the Deutsche Forschungsgemeinschaft (DFG, grant DU 1095/2-1), the Fonds der Chemischen Industrie
(FCI, Sachkostenzuschuss) and the Max Planck Society for financial support. B.S. is grateful for a doctoral fellowship of the Studienstiftung des deutschen Volkes.

\section{Notes and references}

1 V. K. Sharma, P. Rungta and A. K. Prasad, RSC Adv., 2014, 4, 16618-16631.

2 A. J. A. Cobb, Org. Biomol. Chem., 2007, 5, 3260-3275.

3 G. F. Deleavey and M. J. Damha, Chem. Biol., 2012, 19, 937-954.

4 F. H. Westheimer, Science, 1987, 235, 1173-1178.

5 Z. Huang and S. A. Benner, J. Org. Chem., 2002, 67, 3996-4013.

6 A. H. El-Sagheer and T. Brown, J. Am. Chem. Soc., 2009, 131, 3958-3964.

7 J. Lebreton, A. De Mesmaeker, A. Waldner, V. Fritsch, R. M. Wolf and S. M. Freier, Tetrahedron Lett., 1993, 34, 6383-6386.

8 A. De Mesmaeker, A. Waldner, J. Lebreton, P. Hoffmann, V. Fritsch, R. M. Wolf and S. M. Freier, Synlett, 1993, 733-736.

9 A. De Mesmaeker, A. Waldner, J. Lebreton, P. Hoffmann, V. Fritsch, R. M. Wolf and S. M. Freier, Angew. Chem., Int. Ed. Engl., 1994, 33, 226-229.

10 C. Selvam, S. Thomas, J. Abbott, S. D. Kennedy and E. Rozners, Angew. Chem., Int. Ed., 2011, 50, 2068-2070.

11 P. E. Nielsen, M. Egholm, R. H. Berg and O. Buchardt, Science, 1991, 254, 1497-1500.

12 Z. V. Zhilina, A. J. Ziemba and S. W. Ebbinghaus, Curr. Top. Med. Chem., 2005, 5, 1119-1131.

13 J. K. Strauss, C. Roberts, M. G. Nelson, C. Switzer and L. J. Maher III, Proc. Natl. Acad. Sci. U. S. A., 1996, 93, 9515-9520.

14 T. P. Prakash, A. M. Kawasaki, E. A. Lesnik, N. Sioufi and M. Manoharan, Tetrahedron, 2003, 59, 7413-7422.

15 M. Prhavc, T. P. Prakash, G. Minasov, P. D. Cook, M. Egli and M. Manoharan, Org. Lett., 2003, 5, 2017-2020.

16 M. L. Jain, P. Y. Bruice, I. E. Szabo and T. C. Bruice, Chem. Rev., 2012, 112, 1284-1309.

17 S. A. Benner and D. Hutter, Bioorg. Chem., 2002, 30, 62-80.

18 S. A. Benner, Acc. Chem. Res., 2004, 37, 784-797.

19 A. Blasko, R. O. Dempcy, E. E. Minyat and T. C. Bruice, J. Am. Chem. Soc., 1996, 118, 7892-7899.

20 D. P. Arya and T. C. Bruice, J. Am. Chem. Soc., 1998, 120, 6619-6620.

21 D. A. Barawkar and T. C. Bruice, Proc. Natl. Acad. Sci. U. S. A., 1998, 95, 11047-11052.

22 H. Challa and T. C. Bruice, Bioorg. Med. Chem. Lett., 2001, 11, 2423-2427.

23 R. L. Letsinger, C. N. Singman, G. Histand and M. Salunkhe, J. Am. Chem. Soc., 1988, 110, 4470-4471.

24 L. A. McDonald, L. R. Barbieri, F. T. Carter, E. Lenoy, J. Lotvin, P. J. Petersen, M. Siegel, F. Singh and R. T. Williamson, J. Am. Chem. Soc., 2002, 124, 10260-10261.

25 A. P. Spork, S. Koppermann and C. Ducho, Synlett, 2009, 2503-2507.

26 A. P. Spork, S. Koppermann, B. Dittrich, R. Herbst-Irmer and C. Ducho, Tetrahedron: Asymmetry, 2010, 21, 763-766.

27 A. P. Spork and C. Ducho, Synlett, 2013, 343-346.

28 A. P. Spork and C. Ducho, Org. Biomol. Chem., 2010, 8, 2323-2326.

29 A. P. Spork, D. Wiegmann, M. Granitzka, D. Stalke and C. Ducho, J. Org. Chem., 2011, 76, 10083-10098.

30 J.-L. Mergny and L. Lacroix, Oligonucleotides, 2003, 13, 515-537. 